\title{
On the connectedness of the branch loci of moduli spaces of orientable Klein surfaces
}

\author{
Antonio F. Costa, Milagros Izquierdo and Ana M. Porto
}

Linköping University Post Print

Tweet

N.B.: When citing this work, cite the original article.

The original publication is available at www.springerlink.com:

Antonio F. Costa, Milagros Izquierdo and Ana M. Porto, On the connectedness of the branch loci of moduli spaces of orientable Klein surfaces, 2014, Geometriae Dedicata, 1-18.

http://dx.doi.org/10.1007/s10711-014-9983-1

Copyright: Springer Verlag (Germany)

http://www.springerlink.com/?MUD=MP

Postprint available at: Linköping University Electronic Press

http://urn.kb.se/resolve?urn=urn:nbn:se:liu:diva-113695 


\title{
On the connectedness of the branch loci of moduli spaces of orientable Klein surfaces
}

\author{
Antonio F. Costa* \\ Departamento de Matematicas \\ UNED \\ 28040 Madrid, Spain \\ acosta@mat.uned.es
}

\author{
Milagros Izquierdo ${ }^{\dagger}$ \\ Matematiska institutionen \\ Linköpings universitet \\ 58183 Linköping, Sweden \\ miizq@mai.liu.se
}

\author{
Ana M. Porto ${ }^{\ddagger}$ \\ Departamento de Matematicas \\ UNED \\ 28040 Madrid, Spain \\ asilva@mat.uned.es
}

April 12, 2012

2010 Mathematics Subject Classification: 30F50, 30F10, $14 \mathrm{H} 37$.

Abstract. Let $\mathcal{M}_{(g,+, k)}^{K}$ be the moduli space of orientable Klein surfaces of genus $g$ with $k$ boundary components (see [AG], [N2]). The space $\mathcal{M}_{(g,+, k)}^{K}$ has a natural orbifold structure with singular locus $\mathcal{B}_{t}^{K}$ consisting of symmetric Klein surfaces. We prove that $\mathcal{B}_{(g,+, k)}^{K}$ is connected for every $k$, and in particular we give an alternative proof to the one in [BCIP], of the connectedness of $\mathcal{B}_{(g,+, 0)}^{K}$.

\section{Introduction}

The concept of Klein surface was introduced by Felix Klein in the last chapter of his book "On Riemann's Theory of Algebraic Functions and their Integrals" (1882). A Klein surface is a surface with a dianalytic structure and here we are concerned with compact surfaces. For dianalytic structure and Klein surfaces see [AG], [BEGG] or [N2]. Topologically (compact) Klein surfaces are surfaces which might be nonorientable and might have boundary. Klein surfaces are important in the study of

${ }^{*}$ Partially supported by MTM2011-23092.

${ }^{\dagger}$ Work done during a visit to the Institut Mittag-Leffler (Djursholm, Sweden).

${ }^{\ddagger}$ Partially supported by MTM2011-23092. 
real algebraic curves (see $[\mathrm{G}],[\mathrm{CG}],[\mathrm{N} 2]$ ) and in the development of topological field theory $([\mathrm{AN}])$.

The deformations of Riemann surfaces is the cause of the introduction of the moduli space $\mathcal{M}_{g}$ consisting of the complex structures on (orientable) compact surfaces of genus $g$. This space has called constant attention of mathematicians and theoretical physicist due to the connections between such space and string theory ([Na]). As for Riemann surfaces there is as well the moduli space $\mathcal{M}_{t}^{K}$ for the dianalytic structures on surfaces with fixed topological type $t=(h, \pm, k)$, where $h$ is the genus, the sign \pm determines the orientability and $k$ is the number of connected components of the boundary. The spaces $\mathcal{M}_{t}^{K}$ have similarities and differences with $\mathcal{M}_{g}$. For instance $\mathcal{M}_{g}$ is a complex orbifold of (complex) dimension $3 g-3$ but for the topological type $t=(h, \pm, k), \mathcal{M}_{t}^{K}$ is an orbifold with universal covering of (real) dimension $3(\varepsilon h+k-1)-3$, where $\varepsilon$ is either 2 if the sign in the topological type $t$ is + or 1 if the sign is - .

The singular sets $\mathcal{B}_{g}$ and $\mathcal{B}_{t}^{K}$ of the moduli space of Riemann and respectively Klein surfaces are called the branch loci of such orbifolds and consist of the surfaces having no trivial automorphisms, i.e. having symmetries. The fact that $\mathcal{B}_{g}$ or $\mathcal{B}_{t}^{K}$ is connected means that a symmetric surface can be continuously deformed to any other symmetric surface preserving the symmetry property along the whole path of deformation. Recently, the connectedness of $\mathcal{B}_{g}$ has been studied and, for instance, it is shown that $\mathcal{B}_{4}$ is connected [CI2] and $\mathcal{B}_{g}$ for $g \geq 60$ is always disconnected see [CI3] (see also [BI]).

For the case of $\mathcal{B}_{t}^{K}$ some results are known, for instance $\mathcal{B}_{(h,-, 0)}^{K}$ is connected for $h \leq 5[\mathrm{BEM}]$ and in [BCIP] using [Se] (see also [BSS]) is proved that $\mathcal{B}_{(h,+, 0)}^{K}$ is connected. In this paper we obtain that $\mathcal{B}_{(h,+, k)}^{K}$ is connected for every $k$, and in particular we get a proof of the connectedness of $\mathcal{B}_{(h,+, 0)}^{K}$ without the use of [BSS]. The proof of the main result is based in the existence of Klein surfaces having two automorphisms with some given topological types and these are the lemmae in Section 5. In the Section 6 we give the proof of the main result which is a consequence of results in Section 5 together with the fact that the set of points in $\mathcal{M}_{t}^{K}$ corresponding to surfaces with an automorphism with a fixed topological type is connected (this fact is a consequence of [MS], see Section 4).

Similar techniques can be used on the branch loci of the moduli space of nonorientable Klein surfaces, yielding identical main result; they will appear elsewhere.

\section{Klein surfaces and non-euclidean crystallographic groups}

A Klein surface $X$ is a compact surface (may be non-orientable and with boundary) endowed with a dianalytic structure, where a dianalytic structure is a class of atlases where the transition maps are analytic or anti-analytic maps of $\mathbb{C}$. The topological 
type of $X$ is given by $t=(h, \pm, k)$ where $h$ is the genus, + if $X$ is orientable and - if $X$ is non-orientable and $k$ is the number of connected components of the boundary.

A non-Euclidean crystallographic group or NEC group $\Gamma$ is a discrete subgroup of the group $A u t^{ \pm}(\mathbb{D})$ of conformal and anticonformal automorphisms of the unit disc $\mathbb{D}$ of $\mathbb{C}$ and in this paper we shall assume that the orbit space $\mathbb{D} / \Gamma$ is compact. If the NEC group $\Gamma$ does not contain any orientation-reversing automorphism of $\mathbb{D}$, then we say that $\Gamma$ is a Fuchsian group.

The so called canonical presentation for NEC groups first appeared in [W] and their structure was clarified by the introduction of signatures in $[\mathrm{M}]$ (see also [Si]).

Given an NEC group $\Gamma$, the subgroup of $\Gamma$ consisting of the orientation-preserving elements is called the canonical Fuchsian subgroup of $\Gamma$. The algebraic structure of $\Gamma$ and the geometric and topological structure of the quotient orbifold $\mathbb{D} / \Gamma$ are given by the signature:

$$
s(\Gamma)=\left(h ; \pm ;\left[m_{1}, \ldots, m_{r}\right] ;\left\{\left(n_{1,1}, \ldots, n_{1, r_{1}}\right), \ldots,\left(n_{k, 1}, \ldots, n_{k, r_{k}}\right)\right\}\right) .
$$

The orbit space $\mathbb{D} / \Gamma$ is an orbifold with underlying surface of genus $h$, having $r \geq 0$ cone points and $k$ boundary components of the underlying surface, each with $r_{i} \geq 0$ corner points, $i=1, \ldots, k$. The signs " + " and " $-"$ correspond to orientable and non-orientable orbifolds respectively. The integers $m_{i}$ are called the proper periods of $\Gamma$ and they are the orders of the cone points of $\mathbb{D} / \Gamma$. The brackets $\left(n_{i, 1}, \ldots, n_{i, r_{i}}\right)$ are the period cycles of $\Gamma$. The integers $n_{i, j}$ are the link periods of $\Gamma$ and the orders of the corner points of $\mathbb{D} / \Gamma$. The group $\Gamma$ is isomorphic to the fundamental group of the orbifold $\mathbb{D} / \Gamma$.

A group $\Gamma$ with signature (1) has a canonical presentation with four types of generators (called canonical generators):

1. Hyperbolic generators: $a_{1}, b_{1}, \ldots, a_{h}, b_{h}$ if $\mathbb{D} / \Gamma$ is orientable; or glide reflection generators: $d_{1}, \ldots, d_{h}$ if $\mathbb{D} / \Gamma$ is non-orientable,

2. Elliptic generators: $x_{1}, \ldots, x_{r}$,

3. Connecting generators (hyperbolic or elliptic transformations):

$$
e_{1}, \ldots, e_{k},
$$

4. Reflection generators: $c_{i, j}, 1 \leq i \leq k, 1 \leq j \leq r_{i}+1$.

And relators:

1. $x_{i}^{m_{i}}, i=1, \ldots, r$,

2. $c_{i, j}^{2}$,

3. $\left(c_{i, j-1} c_{i, j}\right)^{n_{i, j}}, j=1, \ldots, r_{i}$,

4. $e_{i}^{-1} c_{i, r_{i}} e_{i}^{-1} c_{i, 0}, i=1, \ldots, k$,

5. The long relation:

$$
x_{1} \ldots x_{r} e_{1} \ldots e_{k} a_{1} b_{1} a_{1}^{-1} b_{1}^{-1} \ldots a_{h} b_{h} a_{h}^{-1} b_{h}^{-1} \text { or } x_{1} \ldots x_{r} e_{1} \ldots e_{k} d_{1}^{2} \ldots d_{h}^{2},
$$


according to whether $\mathbb{D} / \Gamma$ is orientable or not.

The hyperbolic area of the orbifold $\mathbb{D} / \Gamma$ coincides with the hyperbolic area of an arbitrary fundamental region of $\Gamma$ and equals:

$$
\mu(\Gamma)=2 \pi\left(\varepsilon h-2+k+\sum_{i=1}^{r}\left(1-\frac{1}{m_{i}}\right)+\frac{1}{2} \sum_{i=1}^{k} \sum_{j=1}^{r_{i}}\left(1-\frac{1}{n_{i, j}}\right)\right),
$$

where $\varepsilon=2$ if there is a $"+^{\prime \prime}$ sign and $\varepsilon=1$ otherwise. If $\Gamma^{\prime}$ is a subgroup of $\Gamma$ of finite index then $\Gamma^{\prime}$ is an NEC group and the following Riemann-Hurwitz formula holds:

$$
\left[\Gamma: \Gamma^{\prime}\right]=\mu\left(\Gamma^{\prime}\right) / \mu(\Gamma) .
$$

An NEC or Fuchsian group $\Gamma$ without elliptic elements is called an NEC or Fuchsian surface group and it has signature $(h ; \pm ;[-],\{(-), \ldots . .,(-)\})$. Given a Klein surface $X$ then $X$ can be represented as the orbit space $X=\mathbb{D} / \Gamma$, with $\Gamma$ an NEC surface group. If a finite group $G$ is isomorphic to a group of automorphisms of $X$ then there exists an NEC group $\Delta$ and an epimorphism $\theta: \Delta \rightarrow G$ with $\operatorname{ker}(\theta)=\Gamma$. The NEC group $\Delta$ is the lifting of $G$ to the universal covering $\pi: \mathbb{D} \rightarrow$ $\mathbb{D} / \Gamma$.

\section{Topological classification of automorphisms of orientable Klein sur- faces}

Two automorphisms $f$ and $g$ of a Klein surface $X$ are topologically equivalent if $f$ and $g$ are conjugated by a homeomorphism of $X$. The topological types of automorphisms are the topological equivalence classes. The topological types of automorphisms are described using topological invariants (see [BCNS], [Y] and $[\mathrm{C}])$. First we shall present the topological types of primer order automorphisms on orientable Klein surfaces.

Assume that $X$ is an orientable Klein surface and let $\varphi: X \rightarrow X$ be an orientation preserving, order $p$ automorphism; where $p$ is a prime. The topological type of $\varphi$ is given by the rotation indices for the fixed points of $\varphi$ and the rotation angles of setwise invariant boundary components. If $\varphi$ leaves $r$ fixed points and left $s$ setwise invariant boundary components, the topological type of $\varphi$ is described by the following data $\theta=\left(p ;+;\left\{n_{1}, \ldots, n_{r}\right\},\left\{m_{1}, \ldots, m_{s}\right\}\right)$ where $1 \leq n_{i}, m_{i} \leq p-1$. The $n_{i}$ (respectively $m_{i}$ ) means that there is a fixed point of $\varphi$ (respectively a boundary component of $X$ ) where locally $\varphi$ acts topologically as a rotation with angle $2 \pi n_{i} / p$ (resp. $2 \pi m_{i} / p$ ). By using uniformization by NEC groups $X$ can be uniformized by a group $\Gamma$ with signature

$$
(g ;+;[-],\{(-), . k .,(-)\})
$$


and the fact of admitting an automorphism of topological type

$$
\theta=\left(p ;+;\left\{n_{1}, \ldots, n_{r}\right\},\left\{m_{1}, \ldots, m_{b}\right\}\right)
$$

implies that there is an NEC group $\Delta$ with signature

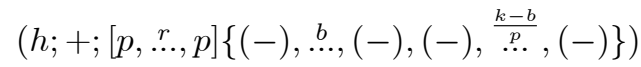

an epimorphism $\omega_{\theta}: \Delta \rightarrow C_{p}=\langle\alpha\rangle$ such that $\Gamma=\operatorname{ker} \omega_{\theta}$ and if $a_{i}, b_{i}, x_{j}, c_{l}, e_{l}$ are a set of canonical generators of $\Delta$, must be:

$$
\omega_{\theta}\left(x_{j}\right)=\alpha^{n_{j}}, \omega_{\theta}\left(e_{l}\right)=\alpha^{m_{j}}, \omega_{\theta}\left(e_{w}\right)=1 \text {, for } w>b
$$

Note that for Riemann-Hurwitz formula $p$ divides $k-b$.

Assume that $X$ is an orientable Klein surface and let $\iota: X \rightarrow X$ be an orientation reversing involution. The topological invariants for $\iota$ are mainly related with Fix $(\iota)$. The set Fix $(\iota)$ consists of:

(a) a finite number $q$ of simple closed curves that we shall call ovals.

(b) a finite number $t$ of chains, which we define now. A chain of length $2 l$ is a set $C$ of $l$ disjoint arcs properly embedded in $X$ (i. e. the ends of each component of $C$ are in the boundary of $X$ ) such that for each boundary component $B$ of $X$, either $C \cap B=\varnothing$ or $C \cap B$ consists of two distinct points.

The extra information that we shall need to determine $\iota$ up topological equivalence is the orientability of $X /\langle\iota\rangle$, where $\langle\iota\rangle$ is the cyclic group of order two generated by $\iota$.

All the above information can be presented in a symbol

$$
\theta=\left(2 ;-; \pm ; q,\left\{s_{1}, \ldots, s_{t}\right\}\right)
$$

that we shall call the species of the involution, where $q$ is the number of ovals, $t$ is the number of chains and $s_{i}$ is the length of each chain and the second sign + is used if $X /\langle\iota\rangle$ is orientable and the - sign is used if $X /\langle\iota\rangle$ is non-orientable. The species is a complete system of invariants for the topological classification of involutions (see [BCNS], [N1]).

By using uniformization by NEC groups $X$ can be uniformized by a group $\Gamma$ with signature $(g ;+;[-],\{(-), . . k,(-)\})$ and the fact of admitting an involution of topological type $\theta=\left(2 ;-; \pm ; q,\left\{s_{1}, \ldots, s_{t}\right\}\right)$ implies that $\Gamma=\operatorname{ker} \omega_{\theta}$ where $\omega_{\theta}: \Delta \rightarrow$ $C_{2}=\langle\iota\rangle, \Delta$ has signature

$$
\left(h ; \pm ;\left\{(-), . . .,(-),(-), . q .,(-),\left(2, . s_{1}, 2\right), . . .,(2, . . ., 2)\right\}\right),
$$

and if either $a_{i}, b_{i}, e_{j}, c_{i}, c_{i, j}$ or $d_{i}, e_{j}, c_{i}, c_{i, j}$ is a set of canonical generators of $\Delta$, must be:

$$
\begin{aligned}
& \omega_{\theta}\left(a_{i}\right)=\omega_{\theta}\left(b_{i}\right)=\omega_{\theta}\left(e_{j}\right)=1 \text { or } \omega_{\theta}\left(d_{i}\right)=\varphi ; \omega_{\theta}\left(e_{j}\right)=1 \\
& \omega_{\theta}\left(c_{i}\right)=1,1 \leq i \leq l ; \omega_{\theta}\left(c_{j}\right)=\varphi, 1 \leq j \leq b ; \omega_{\theta}\left(c_{i, j}\right)=1, \omega_{\theta}\left(c_{i, j+1}\right)=\varphi .
\end{aligned}
$$




\section{Moduli spaces}

Let $s$ be a signature of NEC groups (1) and $\mathcal{G}$ be an abstract group isomorphic to the NEC groups with signature $s$. We denote by $\mathbf{R}(s)$ the set of monomorphisms $r: \mathcal{G} \rightarrow A u t^{ \pm}(\mathbb{D})$ such that $r(\mathcal{G})$ is an NEC group with signature $s$. The set $\mathbf{R}(s)$ has a natural topology given by the topology of $A u t^{ \pm}(\mathbb{D})$. Two elements $r_{1}$ and $r_{2} \in \mathbf{R}(s)$ are said to be equivalent, $r_{1} \sim r_{2}$, if there exists $g \in A u t^{ \pm}(\mathbb{D})$ such that for each $\gamma \in \mathcal{G}, r_{1}(\gamma)=g r_{2}(\gamma) g^{-1}$. The space of classes $\mathbf{T}(s)=\mathbf{R}(s) / \sim$ is called the Teichmüller space of NEC groups with signature $s$ (see [MS]). The Teichmüller space $\mathbf{T}(s)$ is homeomorphic to $\mathbb{R}^{d(s)}$ where

$$
\left.d(s)=3(\varepsilon h-1+k)-3+\left(2 \sum_{i=1}^{r} m_{i}+\sum_{i=1}^{k} \sum_{j=1}^{s_{i}} n_{i j}\right)\right) .
$$

The modular group $\operatorname{Mod}(\mathcal{G})$ of $\mathcal{G}$ is the quotient $\operatorname{Mod}(\mathcal{G})=\operatorname{Aut}(\mathcal{G}) / \operatorname{Inn}(\mathcal{G})$, where $\operatorname{Inn}(\mathcal{G})$ denotes the inner automorphisms of $\mathcal{G}$. The moduli space for NEC groups with signature $s$ is the quotient $\mathcal{M}_{s}=\mathbf{T}(s) / \operatorname{Mod}(\mathcal{G})$ endowed with the quotient topology. Hence $\mathcal{M}_{s}$ is an orbifold with fundamental orbifold group $\operatorname{Mod}(\mathcal{G})$.

If $s$ is the signature of a surface group uniformizing surfaces of topological type $t$, then we denote by $\mathbf{T}(s)=\mathbf{T}_{t}$ and $\mathcal{M}_{s}=\mathcal{M}_{t}$ the Teichmüller and the moduli space of Klein surfaces of topological type $t$.

Let $\mathcal{G}$ and $\mathcal{G}^{\prime}$ be groups isomorphic to NEC groups with signatures $s$ and $s^{\prime}$ respectively. The inclusion mapping $\alpha: \mathcal{G} \rightarrow \mathcal{G}^{\prime}$ induces an embedding $\mathbf{T}(\alpha)$ : $\mathbf{T}\left(s^{\prime}\right) \rightarrow \mathbf{T}(s)$ defined by $[r] \mapsto[r \circ \alpha]$. See [MS].

If a finite group $G$ is isomorphic to a group of automorphisms of Klein surfaces with topological type $t=(g,+, k)$, then the action of $G$ is determined by an epimorphism $\omega_{\theta}: \mathcal{D} \rightarrow G$, where $\mathcal{D}$ is an abstract group isomorphic to NEC groups with a given signature $s$ and $\operatorname{ker}\left(\omega_{\theta}\right)=\mathcal{G}$ is a abstract group isomorphic to surface groups uniformizing Klein surfaces of topological type $t$. Then there is an inclusion $\alpha: \mathcal{G} \rightarrow \mathcal{D}$ and an embedding $\mathbf{T}(\alpha): \mathbf{T}(s) \rightarrow \mathbf{T}_{t}$. The continuous map $\mathbf{T}(\alpha)$ induces a continuous map $\mathcal{M}_{s} \rightarrow \mathcal{M}_{t}$ and as a consequence:

Remark. The set $\mathcal{B}_{t}^{G, \theta}$ of points in $\mathcal{M}_{t}$ corresponding to surfaces having a group of automorphisms isomorphic to $G$, with a fixed action $\theta$, is a connected set.

\section{Klein surfaces with two automorphisms with some given topological types}

The following series of lemmae will be essential in the proof of the main theorem.

Lemma 1 Let $\theta=\left(p ;+;\left\{w_{1}, \ldots, w_{r}\right\},\left\{v_{1}, \ldots, v_{s}\right\}\right)$ be the topological type of an orientation preserving automorphism $\alpha$ of order $p$, with $r+s>0$, on a Klein 
surface $X$ of topological type $t=(g,+, k)$. Hence there exists a Klein surface $Y$ of topological type $t$ with an automorphism of topological type $\theta$ and an orientation reversing involution with topological type $\left(2 ;-;+; b ;\left\{s_{1}, \ldots, s_{l}\right\}\right)$, with $b+l>0$.

Proof. Let $X /\langle\phi\rangle$ be the orbit Klein surface by the action of $\phi$ on $X$. Using the uniformization theorems, there is a surface NEC group $\Gamma$ with signature

$$
s(\Gamma)=(g ;+;[-] ;\{(-), \ldots,(-)\})
$$

such that $\Gamma$ is a normal subgroup of an NEC group $\Delta$ of signature

$$
s(\Delta)=\left(h ;+;[p, . r ., p] ;\left\{(-), . . . \cdot,(-),(-), \stackrel{m=\frac{k-b}{\cdots}}{\cdots},(-)\right\}\right)
$$

and in such a way that there are Klein surfaces isomorphisms $\lambda: X \rightarrow \mathbb{D} / \Gamma$ and $\mu: X /\langle\alpha\rangle \rightarrow \mathbb{D} / \Delta$ making commutative the following diagram:

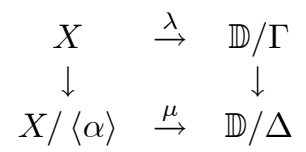

The monodromy epimorphism $\omega_{\theta}: \Delta \rightarrow \Delta / \Gamma \simeq C_{p}=\langle\alpha\rangle$ is determined up topological equivalence by the topological type $\theta$ of the automorphism $\phi$. We can construct a canonical presentation of $\Delta$ :

$$
\begin{gathered}
\left\langle a_{i}, b_{i}, x_{j}, e_{l}, c_{l}, i=1, \ldots, h ; j=1, \ldots, r ; l=1, \ldots, b+m:\right. \\
\left.\prod\left[a_{i}, b_{i}\right] \cdot \prod x_{j} \cdot \prod e_{l}=1 ; c_{l}^{2}=1\right\rangle
\end{gathered}
$$

adapted to the epimorphism $\omega_{\theta}$ in such a way that:

$$
\begin{aligned}
& \omega_{\theta}\left(a_{i}\right)=\omega_{\theta}\left(b_{i}\right)=1, i=1, \ldots, h ; \\
& \omega_{\theta}\left(x_{j}\right)=\alpha^{w_{j}}, j=1, \ldots, r ; w_{j} \in\{1, \ldots, p-1\} ; \\
& \omega_{\theta}\left(e_{l}\right)=\alpha^{v_{l}}, l=1, \ldots, b ; v_{l} \in\{1, \ldots, p-1\} ; \omega_{\theta}\left(e_{l}\right)=1, l=b+1, \ldots, b+m ; \\
& \omega_{\theta}\left(c_{l}\right)=1, l=1, \ldots, b+m .
\end{aligned}
$$

In order to find the surface $Y$ in the statement of the lemma, we consider now a NEC group $\Lambda$ with signature:

$$
s(\Lambda)=\left(d ;+;[-] ;\left\{(p, \stackrel{r}{.}, p, 2,2 b, 2,2,2 m, 2),(-)^{\varepsilon}\right\}\right)
$$

where $d=\frac{h}{2}$ and $\varepsilon=0$, if $h$ is even or $d=\frac{h-1}{2}$ and $\varepsilon=1$, if $h$ is odd.

Let

$$
\begin{gathered}
\left\langle a_{i}, b_{i}, e_{l}, c_{1, u}, c_{2} ; i=1, \ldots, d ; l=1,2 ; u=0, \ldots, r+2 b+2 m:\right. \\
\left.\prod\left[a_{i}, b_{i}\right] \cdot \prod e_{l}=1 ; c_{1 u}^{2}=c_{2}^{2}=1 ; e_{1} c_{1, r+2 b+2 m} e_{1}^{-1} c_{10}=1\right\rangle
\end{gathered}
$$

be a canonical presentation for $\Lambda$ in case $h$ odd and

$$
\begin{gathered}
\left\langle a_{i}, b_{i}, e_{1}, c_{1 u} ; i=1, \ldots, d ; u=0, \ldots, r+2 b+2 m:\right. \\
\left.\prod\left[a_{i}, b_{i}\right] \cdot e_{1}=1 ; c_{1 u}^{2}=1 ; e_{1} c_{1, r+2 b+2 m} e_{1}^{-1} c_{1,0}=1\right\rangle
\end{gathered}
$$


in the case of $h$ even.

Now we consider the epimorphism

$$
\varpi: \Lambda \rightarrow D_{p}=\left\langle\alpha, \sigma: \alpha^{p}=\sigma^{2}=(\alpha \sigma)^{2}=1\right\rangle
$$

defined by:

$$
\begin{aligned}
& \varpi\left(a_{i}\right)=\varpi\left(b_{i}\right)=1 ; i=1, \ldots, d, \\
& \varpi\left(c_{1,0}\right)=\sigma, \varpi\left(c_{1, u}\right)=\alpha^{g_{u}} \sigma, \text { where } g_{u-1}-g_{u}=w_{u} \text { and } g_{0}=0 \\
& \varpi\left(c_{1, r+2 l+1}\right)=1, \varpi\left(c_{1, r+2 l}\right)=\alpha^{f_{l}} \sigma, \text { where } f_{l-1}-f_{l}=v_{l}, l=1, \ldots, b, \text { and } \\
& f_{0}=g_{r} \\
& \varpi\left(c_{1, r+2 l+2 b+1}\right)=1, \varpi\left(c_{1, r+2 b+2 l}\right)=\alpha^{f_{r+2 b}} \sigma .
\end{aligned}
$$

Note that if $h$ is even $f_{r+2 b}=0$ and we define $\varpi\left(e_{1}\right)=1$.

For the case $h$ odd, $\varpi\left(c_{2}\right)=\sigma, \varpi\left(e_{1}\right)$ is determined by the relation

$$
e_{1} c_{1, r+2 b+2 m} e_{1}^{-1} c_{1,0}=1
$$

and $\varpi\left(e_{2}\right)$ is determined by the long relation

$$
\prod\left[a_{i}, b_{i}\right] \cdot \prod e_{l}=1
$$

The surface $Y$ uniformized by $\operatorname{ker} \varpi, Y=\mathbb{D} / \operatorname{ker} \varpi$, admits a conformal automorphism $\alpha$ of topological type $\theta$ (see Section 3 ) that is given by deck transformation of the cyclic covering:

$$
\mathbb{D} / \operatorname{ker} \varpi=Y \rightarrow \mathbb{D} / \varpi^{-1}\langle\alpha\rangle .
$$

As well $Y$ admits an orientation reversing involution $\sigma$

$$
\mathbb{D} / \operatorname{ker} \varpi=Y \rightarrow \mathbb{D} / \varpi^{-1}\langle\sigma\rangle
$$

having non-empty fixed point set (note that the NEC group $\varpi^{-1}\langle\sigma\rangle$ contains reflections).

Lemma 2 Let $\theta=(p ;+;\{-\},\{-\})$ be the topological type of an orientation preserving automorphism $\alpha$ of order $p$, without any fixed point and no invariant boundary component, on a Klein surface $X$ of topological type $t=(g,+, k)$. Hence there exists a Klein surface $Y$ of topological type $t$ with an automorphism of topological type $\theta$ and an orientation preserving involution with topological type $\left(2 ;+;\{1, \ldots, 1\},\left\{1^{\varepsilon}\right\}\right)$, with $x>0$ and $\varepsilon=0$ if $\frac{g-1}{p}$ is even and $\varepsilon=0$ if $\frac{g-1}{p}$ is odd (where $\{0\}$ means $\{-\})$. 
Proof. Let $X /\langle\alpha\rangle$ be the orbit Klein surface. Using the uniformization theorems, there is a surface NEC group $\Gamma$ with signature

$$
s(\Gamma)=(g ;+;[-] ;\{(-), \ldots,(-)\})
$$

such that $\Gamma$ is a normal subgroup of an NEC group $\Delta$ of signature

$$
s(\Delta)=\left(h=\frac{g-1}{p}+1 ;+;[-] ;\{(-), \stackrel{m=k / p}{\cdots},(-)\}\right)
$$

and in such a way that there are Klein surfaces isomorphisms $\lambda: X \rightarrow \mathbb{D} / \Gamma$ and $\mu: X /\langle\alpha\rangle \rightarrow \mathbb{D} / \Delta$ making commutative the following diagram:

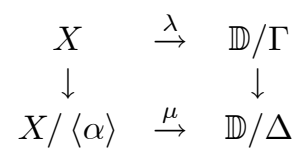

The monodromy epimorphism $\omega_{\theta}: \Delta \rightarrow \Delta / \Gamma \simeq C_{p}=\langle\alpha\rangle$ is determined up topological equivalence by the topological type $\theta$ of the automorphism $\alpha$. We can construct a canonical presentation of $\Delta$ :

$$
\begin{gathered}
\left\langle a_{i}, b_{i}, e_{l}, c_{l}, i=1, \ldots, h ; l=1, \ldots, m:\right. \\
\left.\prod\left[a_{i}, b_{i}\right] \cdot \prod e_{l}=1 ; c_{l}^{2}=1\right\rangle
\end{gathered}
$$

such that the epimorphism $\omega_{\theta}$ has the following form:

$\omega_{\theta}\left(a_{i}\right)=\omega_{\theta}\left(b_{i}\right)=1, i=1, \ldots, h-1 ; \omega_{\theta}\left(a_{h}\right)=\alpha, \omega_{\theta}\left(b_{h}\right)=1$

$\omega_{\theta}\left(e_{l}\right)=1 ; l=1, \ldots, m$;

$\omega_{\theta}\left(c_{l}\right)=1 ; l=1, \ldots, m$.

In order to find the surface $Y$ in the statement of the lemma, we consider now a NEC group $\Lambda$ with signature:

$$
s(\Lambda)=\left(0 ;+;\left[2,{ }^{2 h+2-\varepsilon}, 2\right] ;\left\{(-), \frac{m-\varepsilon}{\stackrel{2}{*}},(-),(-)^{\varepsilon}\right\}\right)
$$

where $\varepsilon=0$, if $h$ is even and $\varepsilon=1$, if $m$ is odd.

Let

$$
\begin{gathered}
\left\langle x_{j}, e_{l}, c_{l} ; j=1, \ldots, 2 h+2-\varepsilon ; l=1, \ldots, \frac{m-\varepsilon}{2}+\varepsilon:\right. \\
\left.\prod x_{j} \cdot \prod e_{l}=1 ; c_{l}^{2}=1\right\rangle
\end{gathered}
$$

be a canonical presentation for $\Lambda$.

Now we consider the epimorphism

$$
\varpi: \Lambda \rightarrow D_{p}=\left\langle\alpha, \sigma: \alpha^{p}=\sigma^{2}=(\alpha \sigma)^{2}=1\right\rangle
$$

defined by:

$$
\begin{aligned}
& \varpi\left(x_{j}\right)=\sigma ; j=1, \ldots, 2 h ; \varpi\left(x_{j}\right)=\alpha \sigma ; j=2 h+1,2 h+2-\varepsilon \\
& \varpi\left(c_{l}\right)=1 ; l=1, \ldots, \frac{m-\varepsilon}{2}+\varepsilon, \\
& \varpi\left(e_{l}\right)=1 ; l=1, \ldots, \frac{m-\varepsilon}{2}, \text { if } \varepsilon=1, \varpi\left(e_{\frac{m-\varepsilon}{2}+1}\right)=\alpha \sigma .
\end{aligned}
$$


The surface $Y$ uniformized by $\operatorname{ker} \varpi, Y=\mathbb{D} / \operatorname{ker} \varpi$, admits a conformal automorphism of order $p$ of topological type $\theta$ that is given by deck transformation of the cyclic covering:

$$
\mathbb{D} / \operatorname{ker} \varpi=Y \rightarrow \mathbb{D} / \varpi^{-1}\langle\alpha\rangle .
$$

As well $S$ admits an orientation preserving involution

$$
\mathbb{D} / \operatorname{ker} \varpi=Y \rightarrow \mathbb{D} / \varpi^{-1}\langle\sigma\rangle
$$

having non-empty fixed point set.

Lemma 3 Let $\theta=\left(2 ;-; \pm ; q ;\left\{s_{1}, \ldots, s_{t}\right\}\right)$ be the topological type of an orientation reversing involution $\alpha$ on a Klein surface $X$ of topological type $t=(g,+, k)$. Hence there exists a Klein surface $Y$ of topological type $t$ with an orientation reversing involution of topological type $\theta$ and an orientation preserving involution.

Proof. Let $X /\langle\alpha\rangle$ be the orbit Klein surface. Using the uniformization theorems, there is a surface NEC group $\Gamma$ with signature

$$
s(\Gamma)=(g ;+;[-] ;\{(-), \ldots,(-)\})
$$

such that $\Gamma$ is a normal subgroup of an NEC group $\Delta$ of signature

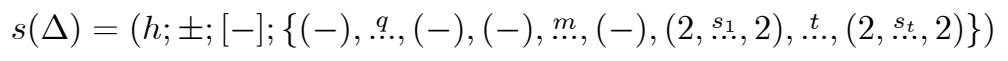

in such a way that there are Klein surfaces isomorphisms $\lambda: X \rightarrow \mathbb{D} / \Gamma$ and $\mu$ : $X /\langle\alpha\rangle \rightarrow \mathbb{D} / \Delta$ making commutative the following diagram:

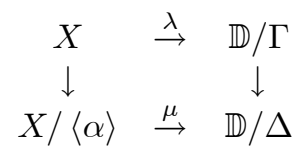

The monodromy epimorphism $\omega_{\theta}: \Delta \rightarrow \Delta / \Gamma \simeq C_{2}=\langle\alpha\rangle$ is determined up topological equivalence by the topological type $\theta$ of the involution $\alpha$. We can construct a presentation of $\Delta$ :

$$
\begin{gathered}
\left\langle\begin{array}{c}
a_{i}, b_{i}, e_{l}, c_{l}, c_{j w}, i=1, \ldots, h ; l=1, \ldots, q+m+t ; \\
j=q+m+1, \ldots, q+m+t ; w=0, \ldots, t_{j}
\end{array}\right. \\
\left.\prod\left[a_{i}, b_{i}\right] \cdot \prod e_{l}=1 ; c_{l}^{2}=c_{j w}^{2}=1 ;\left(c_{j w} c_{j w+1}\right)^{2}=1 ; e_{j} c_{j, t_{j}} e_{j}^{-1} c_{j, 0}=1\right\rangle
\end{gathered}
$$

or

$$
\begin{gathered}
\left\langle\begin{array}{c}
d_{i}, e_{l}, c_{l}, c_{j w}, i=1, \ldots, h ; l=1, \ldots, q+m+t ; \\
j=q+m+1, \ldots, q+m+t ; w=0, \ldots, t_{j}
\end{array} \quad\right. \\
\left.\prod d_{i}^{2} \cdot \prod e_{l}=1 ; c_{l}^{2}=c_{j w}^{2}=1 ;\left(c_{j w} c_{j w+1}\right)^{2}=1 ; e_{j} c_{j, t_{j}} e_{j}^{-1} c_{j, 0}=1\right\rangle
\end{gathered}
$$

depending on the sign in $s(\Delta)$, and such that the epimorphism $\omega_{\theta}$ has the following form: 
$\omega_{\theta}\left(a_{i}\right)=\omega_{\theta}\left(b_{i}\right)=1, i=1, \ldots, h$ if the sign is + and $\omega_{\theta}\left(d_{i}\right)=\alpha, i=1, \ldots, h$ if the sign is - ;

$\omega_{\theta}\left(e_{l}\right)=1, l=1, \ldots, q+m$

$\omega_{\theta}\left(c_{l}\right)=\alpha, l=1, \ldots, q ; \omega_{\theta}\left(c_{l}\right)=1, l=q+1, \ldots, q+m ;$

$\omega_{\theta}\left(c_{j, 2 i}\right)=\alpha, 0 \leq i \leq s_{j} / 2, j=q+m+1, \ldots, q+m+t$,

$\omega_{\theta}\left(c_{j, 2 i+1}\right)=1,0 \leq i \leq s_{j} / 2-1, j=q+m+1, \ldots, q+m+t$.

By the construction of $\omega, 2 m+\sum \frac{s_{j}}{2}=k$.

In order to find the surface $Y$ in the statement of the lemma, we consider now an NEC group $\Lambda$ with signature:

$$
s(\Lambda)=\left(d ;-;\left[2^{\varepsilon}\right] ;\left\{\left(2,2 b, 2,2,2 m, \stackrel{2 m}{2}, 2, s_{1} / 2+2,2, \ldots, 2,{ }^{s_{t} / 2+2}, 2\right)\right\}\right)
$$

where $d=h, \varepsilon=0$ if the sign in $s(\Delta)$ is $+; d=h / 2, \varepsilon=0$ if $h$ is even and the sign in $s(\Delta)$ is - and $d=\frac{h-1}{2}, \varepsilon=1$ if $h$ is odd and the sign in $s(\Delta)$ is -.

Let

$$
\begin{gathered}
\left\langle d_{i}, x, e, c_{j} ; i=1, \ldots, d ; j=0, \ldots, 2 m+2 b+\sum\left(s_{j} / 2+2\right):\right. \\
\left.\prod d_{i}^{2} \cdot x^{\varepsilon} \cdot e=1 ; x^{\varepsilon+1}=1 ; c_{j}^{2}=1 ;\left(c_{j} c_{j+1}\right)^{2}=1 ; e c_{2 m+2 b+\sum s_{j} / 2+2} e^{-1} c_{0}=1\right\rangle
\end{gathered}
$$

be a canonical presentation for $\Lambda$.

Now we consider the epimorphism

$$
\varpi: \Lambda \rightarrow D_{2}=\left\langle\alpha, \sigma: \alpha^{2}=\sigma^{2}=(\alpha \sigma)^{2}=1\right\rangle
$$

defined by:

$$
\begin{aligned}
& \varpi\left(d_{i}\right)=\alpha ; i=1, \ldots, d, \\
& \text { if } \varepsilon=1, \varpi(x)=\alpha \sigma, \varpi(e)=\alpha \sigma, \text { if } \varepsilon=0, \varpi(e)=1, \\
& \varpi\left(c_{2 j}\right)=\sigma, \varpi\left(c_{2 j+1}\right)=\alpha, \text { where } j=0, \ldots, q-1, \\
& \varpi\left(c_{2 m}\right)=\sigma, \\
& \varpi\left(c_{2 m+2 j}\right)=\sigma, \varpi\left(c_{2 m+2 j+1}\right)=1 \text { where } j=0, \ldots, m-1 \\
& \varpi\left(c_{2 m+2 b}\right)=\sigma, \\
& \varpi\left(c_{2 m+2 j+\sum s_{i} / 2+2 j+1}\right)=\alpha, \varpi\left(c_{2 m+2 j+\sum s_{i} / 2+2 j}\right)=1, \\
& \varpi\left(c_{2 m+2 j+\sum s_{i} / 2}\right)=\sigma .
\end{aligned}
$$

The surface $Y$ uniformized by ker $\varpi$ admits an orientation reversing involution of topological type $\theta$ that is given by deck transformation of the cyclic covering:

$$
\mathbb{D} / \operatorname{ker} \varpi=Y \rightarrow \mathbb{D} / \varpi^{-1}\langle\alpha\rangle .
$$

As well $Y$ admits an orientation reversing involution

$$
\mathbb{D} / \operatorname{ker} \varpi=Y \rightarrow \mathbb{D} / \varpi^{-1}\langle\sigma \alpha\rangle
$$


Lemma 4 Let $\theta=(2 ;+;\{1, \stackrel{r}{.}, 1\},\{1, . . ., ., 1\})$ be the topological type of an orientation preserving involution $\alpha$, with $r+b>0$, on a Klein surface $X$ of topological type $t=(g,+, k)$ with $k$ even. Hence there exists a Klein surface $Y$ of topological type $t$ with an orientation preserving involution of topological type $\theta$ and an orientation reversing involution with empty fixed point set, i.e. topological type $(2 ;-;-; 0 ;\{-\})$.

Proof. Using the uniformization theorems, there is a surface NEC group $\Gamma$ with signature

$$
s(\Gamma)=(g ;+;[-] ;\{(-), \ldots,(-)\})
$$

such that $\Gamma$ is a normal subgroup of an NEC group $\Delta$ of signature

$$
s(\Delta)=(h ;+;[2, \underline{r}, 2] ;\{(-), . . .,(-),(-), \underline{m},(-)\})
$$

in such a way that there are Klein surfaces isomorphisms $\lambda: X \rightarrow \mathbb{D} / \Gamma$ and $\mu$ : $X /\langle\alpha\rangle \rightarrow \mathbb{D} / \Delta$ making commutative the following diagram:

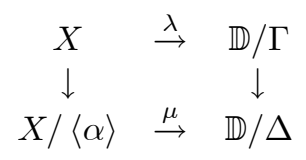

The monodromy epimorphism $\omega_{\theta}: \Delta \rightarrow \Delta / \Gamma \simeq C_{2}=\langle\alpha\rangle$ is determined up topological equivalence by the topological type $\theta$ of the involution $\alpha$. We can construct a presentation of $\Delta$ :

$$
\begin{gathered}
\left\langle a_{i}, b_{i}, x_{j}, e_{l}, c_{l}, i=1, \ldots, h ; j=1, \ldots, r ; l=1, \ldots, b+m:\right. \\
\left.\prod\left[a_{i}, b_{i}\right] \cdot \prod x_{j} \cdot \prod e_{l}=1 ; x_{j}^{2}=c_{l}^{2}=1\right\rangle
\end{gathered}
$$

such that the epimorphism $\omega_{\theta}$ has the following form:

$$
\begin{aligned}
& \omega_{\theta}\left(a_{i}\right)=\omega_{\theta}\left(b_{i}\right)=1, i=1, \ldots, h ; \\
& \omega_{\theta}\left(x_{j}\right)=\alpha, j=1, \ldots, r ; \\
& \omega_{\theta}\left(e_{l}\right)=\alpha, l=1, \ldots, b ; \\
& \omega_{\theta}\left(e_{l}\right)=1, l=b+1, \ldots, b+m ; \\
& \omega_{\theta}\left(c_{l}\right)=1, l=1, \ldots, b+m .
\end{aligned}
$$

Note that $k=2 m+b$ and $r+b$ is even. Since $k$ is even, then $b$ and $r$ are even.

In order to find the surface $Y$ in the statement of the lemma, we consider now a NEC group $\Lambda$ with signature:

$$
\left.s(\Lambda)=\left(h ;-;[2, \stackrel{r / 2}{.}, 2] ;\left\{(-),{ }^{b / 2+m / 2+1},(-)\right\}\right\}\right)
$$

if $m$ is even and

$$
s(\Lambda)=\left(h ;-;[2, \stackrel{r / 2}{\cdots}, 2] ;\left\{(2,2),(-),{ }^{b / 2+(m-1) / 2},(-)\right\}\right)
$$

if $m$ is odd. 
If $m$ is even, let

$$
\begin{gathered}
\left\langle d_{i}, x_{j}, e_{l}, c_{l} ; i=1, \ldots, h ; j=1, \ldots, r / 2 ; l=1, \ldots, b / 2+m / 2+1:\right. \\
\left.\prod d_{i}^{2} \cdot \prod x_{j} \cdot \prod e_{j}=1 ; x_{j}^{2}=c_{l}^{2}=1\right\rangle
\end{gathered}
$$

be a canonical presentation for $\Lambda$. For the case $m$ odd we consider the presentation:

$$
\begin{gathered}
\left\langle d_{i}, x_{j}, e_{l}, c_{1 w}, c_{v} ; i=1, \ldots, h ; j=1, \ldots, r / 2 ; l=1, \ldots, b / 2+(m-1) / 2+1 ;\right. \\
w=0,1,2 ; v=2, \ldots, b / 2+(m-1) / 2+1: \\
\left.\prod d_{i}^{2} \cdot \prod x_{j} \cdot \prod e_{j}=1 ; x_{j}^{2}=c_{l}^{2}=c_{1 w}=1 ; e_{1} c_{13} e_{1}^{-1} c_{10}=1\right\rangle
\end{gathered}
$$

Now we consider the epimorphism

$$
\varpi: \Lambda \rightarrow D_{2}=\left\langle\alpha, \sigma: \alpha^{2}=\sigma^{2}=(\alpha \sigma)^{2}=1\right\rangle
$$

defined by:

$$
\begin{aligned}
& \varpi\left(d_{i}\right)=\sigma ; i=1, \ldots, h ; \\
& \varpi\left(x_{j}\right)=\alpha ; j=1, \ldots, r / 2 ; \\
& \varpi\left(e_{l}\right)=\alpha ; l=2, \ldots, b / 2+1 ; \\
& \varpi\left(e_{l}\right)=1 ; l=b / 2+2, \ldots, b / 2+m / 2+1 \text { or } b / 2+(m-1) / 2+1 ; \\
& \varpi\left(e_{1}\right)=1 \text { if } r / 2+b / 2 \text { is even and } \varpi\left(e_{1}\right)=\alpha \text { if } r / 2+b / 2 \text { is odd, } \\
& \varpi\left(c_{l}\right)=1 ; l=2, \ldots, b / 2+(m-1) / 2+1 ; \\
& \varpi\left(c_{1}\right)=\sigma, \text { if } m \text { is even and } \varpi\left(c_{10}\right)=\sigma, \varpi\left(c_{11}\right)=1, \varpi\left(c_{12}\right)=\sigma \text { if } m \text { is odd. }
\end{aligned}
$$

The surface $Y=\mathbb{D} /$ ker $\varpi$ admits an orientation preserving involution of topological type $\theta$ that is given by deck transformation of the cyclic covering:

$$
\mathbb{D} / \operatorname{ker} \varpi=Y \rightarrow \mathbb{D} / \varpi^{-1}\langle\alpha\rangle .
$$

As well $Y$ admits an orientation reversing involution

$$
\mathbb{D} / \operatorname{ker} \varpi=Y \rightarrow \mathbb{D} / \varpi^{-1}\langle\sigma \alpha\rangle
$$

having empty fixed point set.

Lemma 5 Let $\theta=(2 ;+;\{1, . ., ., 1\},\{1, . ., ., 1\})$ be the topological type of an orientation preserving involution $\alpha$, with $r+s>0$, on a Klein surface $X$ of topological type $t=(g,+, k)$ with $k$ odd. Hence there exists a Klein surface $Y$ of topological type $t$ with an orientation preserving involution of topological type $\theta$ and an orientation reversing involution with topological type $(2 ;-;-; 0 ;\{1\})$.

Proof. Using the uniformization theorems, there is a surface NEC group $\Gamma$ with signature

$$
s(\Gamma)=(g ;+;[-] ;\{(-), \ldots,(-)\})
$$

such that $\Gamma$ is a normal subgroup of an NEC group $\Delta$ of signature

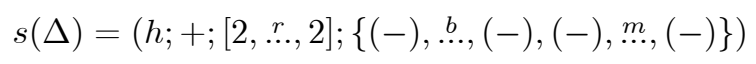


in such a way that there are Klein surfaces isomorphisms $\lambda: X \rightarrow \mathbb{D} / \Gamma$ and $\mu$ : $X /\langle\alpha\rangle \rightarrow \mathbb{D} / \Delta$ making commutative the following diagram:

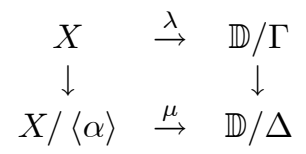

The monodromy epimorphism $\omega_{\theta}: \Delta \rightarrow \Delta / \Gamma \simeq C_{2}=\langle\alpha\rangle$ is determined up topological equivalence by the topological type $\theta$ of the involution $\alpha$. We can construct a presentation of $\Delta$ :

$$
\begin{gathered}
\left\langle a_{i}, b_{i}, x_{j}, e_{l}, c_{l}, i=1, \ldots, h ; j=1, \ldots, r ; l=1, \ldots, b+m:\right. \\
\left.\prod\left[a_{i}, b_{i}\right] \cdot \prod x_{j} \cdot \prod e_{l}=1 ; x_{j}^{2}=c_{l}^{2}=1\right\rangle
\end{gathered}
$$

such that the epimorphism $\omega_{\theta}$ has the following form:

$$
\begin{aligned}
& \omega_{\theta}\left(a_{i}\right)=\omega_{\theta}\left(b_{i}\right)=1, i=1, \ldots, h ; \\
& \omega_{\theta}\left(x_{j}\right)=\alpha, j=1, \ldots, r ; \\
& \omega_{\theta}\left(e_{l}\right)=\alpha, l=1, \ldots, b ; \\
& \omega_{\theta}\left(e_{l}\right)=1, l=b+1, \ldots, b+m ; \\
& \omega_{\theta}\left(c_{l}\right)=1, l=1, \ldots, b+m .
\end{aligned}
$$

Note that $k=2 m+b$ and $r+b$ is even. Since $k$ is odd, then $b$ and $r$ are odd.

In order to find the surface $Y$ in the statement of the lemma, we consider now a NEC group $\Lambda$ with signature:

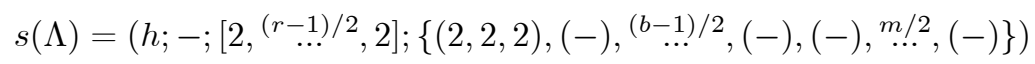

if $m$ is even and

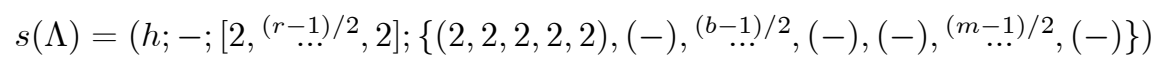

if $m$ is odd.

Let

$$
\begin{gathered}
\left\langle\begin{array}{c}
d_{i}, x_{j}, e_{l},, c_{1 w}, c_{u} ; i=1, \ldots, h ; j=0, \ldots,(r-1) / 2 \\
l=1, \ldots,(b-1) / 2+m / 2+1 ; \\
u=2, \ldots,(b-1) / 2+m / 2+1 ; w=0, . ., 3: \\
u d_{i}^{2} \cdot \prod x_{j} \cdot \prod e_{l}=1 ; x_{j}^{2}=c_{u}^{2}=c_{1 w}^{2}=1 ;\left(c_{l, w} c_{l, w+1}\right)^{2}=1 ; \\
e_{1} c_{l 3} e_{1}^{-1} c_{l 0}=1
\end{array}\right\rangle
\end{gathered}
$$

be a canonical presentation for $\Lambda$ when $m$ is even. For the case $m$ odd we consider:

$$
\begin{gathered}
\left\langle\begin{array}{c}
d_{i}, x_{j}, e_{l}, c_{1 w}, c_{u} ; i=1, \ldots, h ; j=0, \ldots,(r-1) / 2 ; \\
l=1, \ldots,(b-1) / 2+(m-1) / 2+1 ; \\
u=2, \ldots,(b-1) / 2+(m-1) / 2+1 ; w=0, . ., 5: \\
\prod d_{i}^{2} \cdot \prod x_{j} \cdot \prod e_{l}=1 ; x_{j}^{2}=c_{u}^{2}=c_{1 w}^{2}=1 ;\left(c_{l, w} c_{l, w+1}\right)^{2}=1 ; \\
e_{1} c_{l 3} e_{1}^{-1} c_{l 0}=1
\end{array}\right\rangle
\end{gathered}
$$


Now we consider the epimorphism $\varpi: \Lambda \rightarrow D_{2}=\left\langle\alpha, \sigma: \alpha^{2}=\sigma^{2}=(\alpha \sigma)^{2}=1\right\rangle$ defined by:

$$
\begin{aligned}
& \varpi\left(d_{i}\right)=\sigma ; i=1, \ldots, h ; \\
& \varpi\left(x_{j}\right)=\alpha ; j=1, \ldots,(r-1) / 2 ; \\
& \varpi\left(e_{l}\right)=\alpha ; l=2, \ldots,(b-1) / 2+1 ; \\
& \varpi\left(e_{l}\right)=1 ; l=b / 2+2, \ldots,(b-1) / 2+(m-1) / 2+1 ; \\
& \varpi\left(e_{1}\right)=1 \text { if }(r-1) / 2+(b-1) / 2 \text { is even and } \varpi\left(e_{b / 2+m / 2+1}\right)=\alpha \text { if }(r-1) / 2+ \\
& (b-1) / 2 \text { is odd, } \\
& \varpi\left(c_{10}\right)=\sigma, \varpi\left(c_{11}\right)=\sigma \alpha, \varpi\left(c_{12}\right)=1, \varpi\left(c_{13}\right)=\sigma, \text { if } m \text { is even, and } \\
& \varpi\left(c_{10}\right)=\sigma, \varpi\left(c_{11}\right)=\sigma \alpha, \varpi\left(c_{12}\right)=1, \varpi\left(c_{13}\right)=\sigma, \varpi\left(c_{12}\right)=1, \varpi\left(c_{13}\right)=\sigma, \text { if } m
\end{aligned}
$$
is odd,

$$
\varpi\left(c_{u}\right)=1 ; l=2, \ldots, b / 2+m / 2+1 .
$$

The surface $Y=\mathbb{D} /$ ker $\varpi$ admits an orientation preserving involution of topological type $\theta$ that is given by deck transformation of the cyclic covering:

$$
\mathbb{D} / \operatorname{ker} \varpi=Y \rightarrow \mathbb{D} / \varpi^{-1}\langle\alpha\rangle .
$$

As well $Y$ admits an orientation reversing involution

$$
\mathbb{D} / \operatorname{ker} \varpi=Y \rightarrow \mathbb{D} / \varpi^{-1}\langle\sigma \alpha\rangle
$$

with a chain of length 1 as fixed point set and non-orientable quotient space (see $[\mathrm{H}]$ and $[\mathrm{HS}])$.

\section{Connectedness of the branch locus of moduli spaces of orientable Klein surfaces}

Theorem 6 If $t=(g ;+; k)$ with $g \geq 2$, then the branch locus $\mathcal{B}_{t}^{K}$ of the moduli space $\mathcal{M}_{t}^{K}$ of Klein surfaces with topological type $t$ is connected.

Proof. Let $\Theta_{p}^{+}$be set of topological types of actions of orientation preserving automorphisms of prime order $p$ on Klein surfaces of topological type $t$ and $\Theta_{2}^{-}$ be the set of topological types of actions of orientation reversing involutions on Klein surfaces of topological type $t$. If $\theta \in\left(\cup_{p} \Theta_{p}^{+}\right) \cup \Theta_{2}^{-}$we shall denote $\mathcal{B}_{t}^{\theta}$ the set of points in $\mathcal{B}_{t}^{K}$ corresponding to Klein surfaces admitting an automorphism of topological type $\theta$.

Since each group of automorphism of a Klein surface must contain either an automorphism of prime order or an anticonformal involution we have:

$$
\mathcal{B}_{t}^{K}=\bigcup_{\theta \in \cup_{p} \Theta_{p}^{+}} \mathcal{B}_{t}^{\theta} \cup \bigcup_{\theta \in \Theta_{2}^{-}} \mathcal{B}_{t}^{\theta}
$$


As we remark at the end of Section 4 , the $\mathcal{B}_{t}^{\theta}$ are connected sets.

By lemmae 4 and 2, if $k$ is even, the set:

$$
\mathcal{C}=\bigcup_{\theta \in \cup \Theta_{2}^{+}} \mathcal{B}_{t}^{\theta} \cup \mathcal{B}_{t}^{(2 ;-;-; 0 ;\{-\})}
$$

is connected, analogously by lemmae 5 and 2 , if $k$ is odd, the set:

$$
\mathcal{C}=\bigcup_{\theta \in \cup \Theta_{2}^{+}} \mathcal{B}_{t}^{\theta} \cup \mathcal{B}_{t}^{(2 ;-;-; 0 ;\{1\})}
$$

is connected.

Now by lemma 3 :

$$
\mathcal{C} \cup \bigcup_{\theta \in \Theta_{2}^{-}} \mathcal{B}_{t}^{\theta}=\bigcup_{\theta \in \cup \Theta_{2}^{+}} \mathcal{B}_{t}^{\theta} \cup \bigcup_{\theta \in \Theta_{2}^{-}} \mathcal{B}_{t}^{\theta}
$$

is a connected set. And finally by lemmae 1 and 2:

$$
\bigcup_{\theta \in \cup_{p>2} \Theta_{p}^{+}} \mathcal{B}_{t}^{\theta} \cup \underset{\theta \in \cup_{2} \Theta_{2}^{+}}{\bigcup} \mathcal{B}_{t}^{\theta} \cup \bigcup_{\theta \in \Theta_{2}^{-}} \mathcal{B}_{t}^{\theta}=\mathcal{B}_{t}^{K}
$$

is connected.

\section{References}

[AN] Alexeevski, A.; Natanzon, S. Noncommutative two-dimensional topological field theories and Hurwitz numbers for real algebraic curves. Selecta Math. (N.S.) 12 (2006), no. 3-4, 307-377.

[AG] Alling, N. L.; Greenleaf, N., Foundations of the theory of Klein surfaces. Lecture Notes in Mathematics, Vol. 219. Springer-Verlag, BerlinNew York, 1971. ix +117 pp.

[BCIP] Bartolini G.; Costa A. F.; Izquierdo M., Porto, A. M., On the connectedness of the branch locus of the moduli space of Riemann surfaces. Rev. R. Acad. Cienc. Exactas Fís. Nat. Ser. A Math. RACSAM 104 (2010), no. 1, $81-86$.

[BI] Bartolini, G.; Izquierdo, M., On the connectedness of the branch locus of the moduli space of Riemann surfaces of low genus. Proc. Amer. Math. Soc. 140 (2012), no. 1, 35-45, 
[BCNS] Bujalance, E.; Costa, A. F.; Natanzon, S. M.; Singerman, D., Involutions of compact Klein surfaces. Math. Z. 211 (1992), no. 3, 461-478.

[BEGG] Bujalance, E.; Etayo, J. J.; Gamboa, J. M.; Gromadzki, G., Automorphism groups of compact bordered Klein surfaces. A combinatorial approach. Lecture Notes in Mathematics, 1439. Springer-Verlag, Berlin, 1990. xiv $+201 \mathrm{pp}$.

[BEM] Bujalance, E.; Etayo, J. J.; Martínez, E.; Szepietowski, B., On the connectedness of the branch loci of nonorientable unbordered Klein surfaces of low genus. Preprint 2011.

[BSS] Buser, P.; Seppälä, M.; Silhol, R., Triangulations and moduli spaces of Riemann surfaces with group actions. Manuscripta Math. 88 (1995), no. $2,209-224$.

[CG] Cirre, F. J.; Gamboa, J. M. Compact Klein surfaces and real algebraic curves. Topics on Riemann surfaces and Fuchsian groups (Madrid, 1998), 113-131, London Math. Soc. Lecture Note Ser., 287, Cambridge Univ. Press, Cambridge, 2001.

[C] Costa A. F., Classification of the orientation reversing homeomorphisms of finite order of surfaces. Topology Appl. 62 (1995), no. 2, 145-162.

[CI1] Costa, A. F.; Izquierdo, M., On the connectedness of the locus of real Riemann surfaces. Ann. Acad. Sci. Fenn. Math. 27 (2002), no. 2, 341356.

[CI2] Costa A. F.; Izquierdo M., On the connectedness of the branch locus of the moduli space of Riemann surfaces of genus 4. Glasg. Math. J. 52 (2010) no. $2,401-408$.

[CI3] Costa A. F.; Izquierdo M., On the connectivity of the branch locus of Riemann surfaces, Preprint 2011.

[G] Gamboa, J. M., Compact Klein surfaces with boundary viewed as real compact smooth algebraic curves. Mem. Real Acad. Cienc. Exact. Fís. Natur. Madrid 27 (1991), iv+96 pp.

[H] Hoare, A. H. M. Subgroups of N.E.C. groups and finite permutation groups. Quart. J. Math. Oxford Ser. (2) 41 (1990), no. 161, 45-59. 
[HS] Hoare, A. H. M.; Singerman, D., The orientability of subgroups of plane groups. Groups-St. Andrews 1981 (St. Andrews, 1981), pp. 221-227, London Math. Soc. Lecture Note Ser., 71, Cambridge Univ. Press, Cambridge, 1982.

[M] Macbeath, A. M., The classification of non-Euclidean plane crystallographic groups, Can. J. Math. 19 (1966) 1192-1205.

[MS] Macbeath, A. M.; Singerman, D., Spaces of subgroups and Teichmüller space. Proc. London Math. Soc. (3) 31 (1975), no. 2, 211-256.

[Na] Nag, S.: Mathematics in and out of string theory. Topology and Teichmüller spaces (Katinkulta, 1995), 187Đ220, World Sci. Publ., River Edge, NJ, 1996.

[N1] Natanzon, S. M., Topological classification of pairs of commuting antiholomorphic involutions of Riemann surfaces, Russ. Math. Surv. 45 (1986) 159-160.

[N2] Natanzon, S. M., Klein surfaces, Russian Math. Surveys 45 (1990) no. 6, $53-108$.

[N3] Natanzon, S. M., Moduli of Riemann surfaces, real algebraic curves, and their superanalogs. Translated from the 2003 Russian edition by Sergei Lando. Translations of Mathematical Monographs, 225. American Mathematical Society, Providence, RI, 2004. viii+160 pp. ISBN: 0-8218-3594-7.

[Se] Seppälä, M., Real algebraic curves in the moduli space of complex curves. Compositio Math. 74 (1990), no. 3, 259-283.

[Si] Singerman, D., On the structure of non-euclidean crystallographic groups, Proc. Camb. Phil. Soc. 76 (1974) 233-240.

[W] Wilkie, H. C., On non-Euclidean crystallographic groups, Math. Z. 91 (1966) 87-102.

[Y] Yocoyama, K., Complete classification of periodic maps on compact surfaces, Tokyo J. Math. 15 (1992) 247-279. 